Pacific Journal of Mathematics

SOME GENERAL PROPERTIES OF MULTI-VALUED 


\title{
SOME GENERAL PROPERTIES OF MULTI-VALUED FUNCTIONS
}

\author{
RAYMOND E. SMITHSON
}

The object is to determine what theorems for single-valued functions can be extended to which class of multi-valued functions. It is shown that an arc cannot be mapped onto a circle by a continuous, monotone multi-valued function when the image of each point is an arc. On the other hand, the arc can be mapped onto a nonlocally connected space by a monotone, continuous function such that the image of each point is an arc. Characterizations of nonalternating functions analogous to the results in the single-valued theory are obtained, and it is shown that an nonalternating semi-single-valued continuous function on a dendrite is monotone. An analog of the monotone light factorization theorem is obtained for semisingle-valued continuous functions.

Some other results are: an open continuous function with finite images maps a regular curve onto a regular curve, and a continuous function with finite images maps a locally connected, compact space onto a locally connected compact space.

A number of definitions for continuity have been proposed for multivalued or set-valued functions, and Wayman Strother studied the problem of continuity extensively $[10,11,12]$. Also Choquet [2] has studied upper and lower semi-continuous functions. Further, Berge, unlike most authors, allows functions to be multi-valued in [1]. However, much of the work that has been done on set-valued functions has been devoted to the discovery of fixed point theorems ([3], [7] through [9], [11], [13], and [15] through [17]). The purpose of this paper is to investigate properties of multi-valued functions which are similar to the properties of single-valued functions studied in G. T. Whyburn's book, Analytic Topology, [18].

We shall use the following topology on the set of closed subsets of a space $Y$. Let

$$
S(Y)=\{E \subset Y: E \text { is closed and nonempty }\} .
$$

Let $S(Y)$ have the topology used by Michael [6]; i.e., if $V_{1}, \cdots, V_{n}$ are open subsets of $Y$, then the collection $\left\langle V_{1}, \cdots, V_{n}\right\rangle=\{E \in S(Y)$ : $E \cap V_{i} \neq \phi$ for all $i$, and $\left.E \subset \bigcup_{i=1}^{n} V_{i}\right\}$ is a basis for the open sets of $S(Y)$. We shall call this topology the finite topology. This is equivalent to

Received April 3, 1963, and in revised form March 31, 1964. 
the topology used by Strother [3] and Frink [4]. Since we shall be dealing extensively with subspaces of $S(Y)$ we shall use $\left\langle V_{1}, \cdots, V_{n}\right\rangle$ to be either a basic open set in $S(Y)$ or a basic, relatively open set in the appropriate subspace of $S(Y)$. If $\mathscr{V}=\left\{V_{1}, \cdots, V_{n}\right\}$, then set $\langle\mathscr{Y}\rangle=\left\langle V_{1}, \cdots, V_{n}\right\rangle$.

In this paper we shall assume that all spaces are Hausdorff.

Given a set-valued function $F: X \rightarrow Y$ with $F(x)$ closed and nonempty we define the induced function $f$ on $X$ into $S(Y)$ by setting $f(x)=$ $F(x)$ for each $x \in X$. Note that $f$ is single-valued, and $f$ will always denote the function induced by $F$ unless otherwise stated. Also we shall always use upper case letters to denote multi-valued functions.

If $A$ is a subset of $X$, then the symbols $\bar{A}$ and $C l(A)$ are used to denote the closure of $A$, and the symbol $A^{\circ}$ is used to denote the interior of $A$.

Henceforth, we assume that $S(Y)$ has the finite topology, and that $F: X \rightarrow Y$ is, unless otherwise stated, a function such that $F(x)$ is in $S(Y)$ for each $x$ in $X$.

1. Preliminaries. This section will be devoted mainly to gathering known results that are needed in the development of succeeding sections.

Definition. A multi-valued function $F: X \rightarrow Y$ is called continuous in case the induced function $f: X \rightarrow S(Y)$ is continuous.

Notation. If $A \subset X$, then $F(A)=\cup\{F(x): x \in A\}$.

Now we have the following lemmas due to Strother [10].

LEMmA 1.1. A function $F: X \rightarrow Y$ is continuous if and only if statements (1) and (2) hold.

1. If $x_{0} \in X, V$ is open in $Y$, and if $F\left(x_{0}\right) \cap V \neq \phi$, then there exists an open set $U$ of $X$ with $x_{0} \in U$ such that $F(x) \cap V \neq \phi$ for all $x \in U$.

2. If $x_{0} \in X$ and $F\left(x_{0}\right) \subset V$ where $V$ is open in $Y$, then there exists an open set $U$ containing $x_{0}$ such that $F(U) \subset V$.

LEMmA 1.2. Let $Y$ be regular. If $F: X \rightarrow Y$ is continuous, if $\left\{x_{d}\right\}$ is a net in $X$ converging to $x_{0}$, and if $y_{d} \in F\left(x_{d}\right)$ such that $\left\{y_{d}\right\}$ converges to $y_{0}$, then $y_{0} \in F\left(x_{0}\right)$.

Lemma 1.3. Let $F: X \rightarrow Y$ be continuous, and let $X$ and $Y$ be compact. Then $F$ is closed; i.e., $F(A)$ is closed in $Y$ whenever $A$ is closed in $X$. 
We also need the following lemma from Michael [6].

LEMMA 1.4. If $\mathscr{B}$ is a collection of subsets of $Y$ which is disjoint (a subcollection of $S(Y)$ ) and connected in the factor (finite) topology and all (one) of whose elements are (is) connected, then $\cup\{E: E \in \mathscr{P}\}$ is connected.

Set $\mathscr{F}(Y)=\{E \in S(Y) ; E$ finite $\}, \mathscr{C}(Y)=\{E \in S(Y): E$ is compact $\}$ and $\mathscr{F}_{n}(Y)=\{E: E$ has at most $n$ elements $\}$.

Now we can apply the above results to obtain some further lemmas. Lemma 1.5 is a variation of a theorem in Berge [1].

Lemma 1.5. Let $F$ be continuous and onto, and let $X$ be compact. Then $Y$ is compact if and only if $F(x)$ is compact for each $x \in X$.

Proof. Suppose $Y$ is compact; then $F(x)$ closed implies $F(x)$ compact. Suppose that $F(x)$ is compact for each $x$, and let $\mathscr{Y}$ be an open cover of $Y$. Then for each $x$ we obtain a subcover $\mathscr{Y}_{x}$ of $F(x)$, such that $F(x) \cap V \neq \phi$ for all $V \in \mathscr{V}_{x}$. Since $F(x)$ is compact, there is a finite subcover $\mathscr{V}_{x}^{\prime}$ of $F(x)$ in $\mathscr{V}_{x}$, and $F(x) \in\left\langle\mathscr{Y}_{x}^{\prime}\right\rangle$. The collection $\left\{\left\langle\mathscr{V}_{x}^{\prime \prime}\right\rangle: x \in X\right\}$ is an open cover for $f(X)$ in $S(Y)$. Since $f(X)$ is compact, there is a finite subcover, say $\left\langle\mathscr{V}_{1}^{-1}\right\rangle, \cdots,\left\langle\mathscr{V}_{n}^{\prime}\right\rangle$ of $f(X)$; hence the collection $\mathscr{V}_{0}^{n}=\bigcup_{i=1}^{n} \mathscr{Y}_{i}^{\prime}$ is a finite subcover of $Y$ and $\mathscr{V}_{0} \subset \mathscr{V}^{\text {. }}$.

Lemma 1.6. Let $F$ be continuous and $A$ a connected subset of $X$. Then, if $F(x)$ is connected for some $x \in A, F(A)$ is a connected subset of $Y$.

Proof. Since $F$ is continuous, $f(A)$ is connected in $S(Y)$, and for some $x, F(x) \in f(A)$ is connected. So by Lemma 1.4, $F(A)=\cup\{F(x)$ : $x \in A\}$ is connected.

COROLlary 1.7. If $F$ is continuous, if $X$ is connected, and if there is an $x \in X$ such that $F(x)$ is connected, then $F(X)$ is connected. Hence $Y$ is connected if $F$ is onto.

COROLlary 1.8. Let $F$ be continuous. Then $F(A)$ is connected for every connected subset $A$ of $X$ if and only if $F(x)$ is connected for each $x \in X$.

Proof. Since $\{x\}$ is connected, $F(x)$ must be connected by hypothesis. On the other hand, if $A \neq \phi$, then for any $x \in A, F(x)$ is connected. So Lemma 1.6 applies. 
Another result from Michael's paper [6] we need is the following.

LEMMA 1.9. If $A \subset Y$ is closed, the following hold.

1. $\{E \in S(Y): E \subset A\}$ is closed.

2. $\{E \in S(Y): E \cap A \neq \phi\}$ is closed.

COROLlaRY 1.10. If $F$ is continuous, the set $\{x: y \in F(x)\}$ is closed for each $y$.

Proof. The set $\{x: y \in F(x)\}=f^{-1}\{F(x): F(x) \cap\{y\} \neq \phi\}$ and the latter is closed by part 2 of Lemma 1.9.

We call $\{x: y \in F(x)\}$ the inverse of $y$ and write $F^{-1}(y)$. Similarly, for $A \subset Y$ we define

$$
F^{-1}(A)=\{x: F(x) \cap A \neq \phi\} .
$$

Note that if $A$ is closed, so is $F^{-1}(A)$.

Notation. We write $E=A \cup B, A \mid B$ to denote a separation of $E$, and we say that $A$ and $B$ separate $E$.

Note. In general, for $A \subset Y$ we need not have $F\left(F^{-1}(A)\right)=A$. We can generalize a lemma of Whyburn's.

LEMma 1.11. Let $X$ be compact, $Y$ regular, $F: X \rightarrow Y$ continuous, and let $Y_{0} \subset Y$. If $F^{-1}\left(Y_{0}\right)=A \cup B, \quad A \mid B$ with $F(A)$ and $F(B)$ intersecting the same quasi-component $Q$ of $Y_{0}$, then there exists $y_{0} \in Y_{0}$ such that $F^{-1}\left(y_{0}\right)$ intersects $A$ and $B$.

Proof. Let $A_{1}=F(A) \cap Y_{0}$ and $B_{1}=F(B) \cap Y_{0}$. Now, by hypothesis $A_{1} \cap Q \neq \phi$ and $B_{1} \cap Q \neq \phi$. Therefore, $A_{1}$ is not separated from $B_{1}$, so there is a net $\left\{y_{\alpha}\right\}$ in $A_{1}$, say, such that $y_{\alpha} \rightarrow y_{0} \in B_{1}$.

Now let $x_{\alpha} \in F^{-1}\left(y_{\alpha}\right) \cap A$ for each $\alpha$. This defines a net in $F^{-1}\left(A_{1}\right) \cap$ $A$, and since $X$ is compact $\left\{x_{\alpha}\right\}$ has a limit point $x_{0}$ and thus a convergent subnet $x_{\gamma} \rightarrow x_{0}$. By Lemma 1.2, $y_{0} \in F\left(x_{0}\right)$ so $x_{0} \in F^{-1}\left(y_{0}\right)$. Further, $A \mid B$ implies that $x_{0} \mid B$ and so $x_{0} \in A$ or $F^{-1}\left(y_{0}\right) \cap A \neq \phi$. Finally, $y_{0} \in B_{1}$ implies that $F^{-1}\left(y_{0}\right) \cap B \neq \phi$.

Let $X, Y$, and $Z$ be spaces and $F_{1}: X \rightarrow Y, F_{2}: Y \rightarrow Z$ be set-valued functions. The composition function $F=F_{2} \circ F_{1}$ is defined by $F(x)=$ $F_{2}\left(F_{1}(x)\right)$ for each $x \in X$. Note that in this case $F(x)$ may not be a closed set. Also, if $z \in Z$, then $F^{-1}(z)=F_{1}^{-1}\left(F_{2}^{-1}(z)\right)$. Consequently, we write $F^{-1}=F_{1}^{-1} F_{2}^{-1}$. When $X, Y$, and $Z$ are compact we have the following result from [10].

Lemma 1.12. If $F_{1}: X \rightarrow Y$ and $F_{2}: Y \rightarrow Z$ are continuous and 
if $X, Y$, and $Z$ are compact, then $F=F_{2} \circ F_{1}$ is continuous.

Let $F: X \rightarrow Y$ and let $A$ be a subspace of $X$. Then the restriction of $F$ to $A, F \mid A$, is defined by $F \mid A(x)=F(x)$ for all $x \in A$. An immediate consequence of Lemma 1.1 is:

Lemma 1.13. Let $F: X \rightarrow Y$ be continuous and let $A \subset X$. Then the restriction of $F$ to $A$ is continuous.

2. Monotone functions. In this section we generalize the definition of monotone functions and investigate their elementary properties.

Definition. A continuous function $F: X \rightarrow Y$ is called monotone if and only if $F^{-1}(y)$ is connected for each $y \in Y$.

Another generalization of a lemma in Whyburn [18] is:

Lemma 2.1. If $X$ is compact, $Y$ regular, and $F: X \rightarrow Y$ is continuous, then $F$ is monotone if and only if $F^{-1}(A)$ is connected whenever $A$ is a connected subset of $Y$.

Proof. If $F^{-1}(A)$ is connected for each connected set in $Y$, then $F^{-1}(y)$ is connected for each $y \in Y$ and hence $F$ is monotone.

On the other hand, suppose that $F$ is monotone and that $A$ is a connected subset of $Y$. Further, suppose that $F^{-1}(A)=C \cup D$ with $C \mid D$. Both $F(C)$ and $F(D)$ meet $A$, and $A$ is a quasi-component of itself. Thus, by Lemma 1.11, there exists a $y \in A$ such that $F^{-1}(y) \cap$ $C \neq \phi$ and $F^{-1}(y) \cap D \neq \phi$, a contradiction, as $F$ is monotone. Hence $F^{-1}(A)$ is connected.

Whyburn shows the following properties are preserved by monotone, continuous, single-valued functions, the property of being, (1) a unicoherent continuum, (2) a hereditarily locally connected continuum, (3) a regular curve, and (4) a rational curve. However, the following examples show that these properties fail to be preserved by continuous, monotone, multi-valued functions, even when fairly stringent conditions are placed on the set $F(x)$, i.e., we may require $F(x)$ to be a locally connected continuum for each $x \in X$ and have $X$ a locally connected continuum, but still not have $Y$ locally connected. See Example 6 .

Example 1. Let $X$ and $Y$ be any two spaces. Define $F(x)=Y$ for each $x \in X$. Clearly $F$ is continuous and $Y$ need not possess any property that is not shared by all spaces. Thus we see the necessity of placing restrictions on the sets $F(x)$. 
ExAMPLe 2. Let $X$ be the closed interval $[(0,0),(0,1)]$ in the plane, let $Y$ be the circle that is tangent to the $x$-axis at $x=2$ and the line $y=1$ at $(2,1)$. Denote the points of $X$ by their $y$-coordinate, and in $Y$ let the closed arc $(x, y)-(2,0)-\left(x^{\prime}, y\right)$ be denoted by $y y^{\prime}$, where we denote the point $(x, y)$ by $y$ and $\left(x^{\prime}, y\right)$ by $y^{\prime}$. Then define $F$ by $F(0)=(2,0), F(y)=y y^{\prime}, 0<y<1$ and $F(1)=Y$. It is easily seen that $F$ is continuous. In fact $f$ is a homeomorphism and $F$ is monotone. However, $X$ is unicoherent and $Y$ is not.

Example 3. Let $X=[(0,0),(0,1)]$ as above and let $Y$ be the unit square and its interior with corners $(1,0),(2,0),(1,1)$, and $(2,1)$. Let the closed horizontal lines $[(1, y),(2, y)]$ be denoted by $\hat{y}$ where $y$ is the common $y$-coordinate. Again identify the points of $X$ with their $y$-coordinate.

Then let $F(y)=\hat{y}$. Here again $f$ is a homeomorphism. In fact $F$ is monotone and the inverse of a single-valued continuous function of $Y$ onto $X$. Further, $X$ and $F(x)$ are locally connected continua for each $x$. Also, $X$ and $F(x)$ are hereditarily locally connected and hereditarily unicoherent, but $Y$ is neither, and $Y$ is neither rational nor regular, but $X$ is both.

ExAmple 4. Let $X=[0,1]$, and $Y$ the area between and including two concentric circles $C_{0}$ and $C_{1}$. Let $C_{a}, 0 \leqq a \leqq 1$, be the circle that has the same center as $C_{0}$ and $C_{1}$, and with radius $r_{a}=a r_{1}+$ $(1-a) r_{0}$ where $r_{0}, r_{1}$ are the radii of $C_{0}$ and $C_{1}$, respectively. Define $F$ by $F(x)=C_{x}$. Then $F$ is monotone, continuous and $F(x)$ is a locally connected continuum for each $x$, and if $x_{1} \neq x_{2}, F\left(x_{1}\right) \cap F\left(x_{2}\right)=$ $\phi$. Yet $X$ is unicoherent and $Y$ is not.

In Whyburn [18] it is shown that the image of a simple arc under a continuous, monotone transformation is again a simple arc, and similarly for a simple closed curve. However, in the case of multi-valued functions neither of these results holds. Example 5 is a counterexample for the former, and the function that maps each point of the circle onto the entire unit interval serves nicely as a counterexample for the latter. We shall, however, subsequently show that the unit interval cannot be mapped onto the circle by a continuous, monotone, multi-valued function $F$, for which $F(x)$ is a simple arc for each $x$. (Here and in the following $F(x)$ may be degenerate, i.e., a point.)

EXAMPLE 5. Let $I$ be the unit interval. Let $I_{1}, I_{2}$, and $I_{3}$ be copies of $I$. Form $Y$ by erecting $I_{2}$ perpendicular to $I_{1}$ at $1 / 4$ and by erecting $I_{3}$ prependicular to $I_{1}$ at $3 / 4$ (the 0 of $I_{2}$ is identified with $1 / 4$ in $I_{1}$ and the 0 of $I_{3}$ is identified with $3 / 4$ in $I_{1}$ ). Define $F: I \rightarrow Y$ by 
$F(0)=[0,1 / 4] \cup I_{2}, F(1 / 4)=I_{2}, \quad F(1 / 2)=I_{2} \cup[1 / 4,3 / 4] \cup I_{3}, \quad F(3 / 4)=I_{3}$, and $F(1)=I_{3} \cup[3 / 4,1]$ (where intervals are subsets of $I_{1}$ unless otherwise stated). For other points in $I, F$ is defined by ratios. The function $F$ constructed in this manner is monotone and continuous. Also $F(x)$ is an arc for each $x \in I$. Note that the range of $F$ is a space with two branch points and that $F$ is also nonalternating (see $\S 3)$ but not open.

EXAMPLE 6. A construction similar to that of Example 5 can be used to define a continuous, monotone function with $F(x)$ an arc for each $x$ on the unit interval onto the following nonlocally connected planar space. The space consists of the union of the following subsets of the plane: $\{(x, 0): 0 \leqq x \leqq 1\},\{(0, y): 0 \leqq y \leqq 1\}$, and $\{(1 / n, y): n \geqq 2$, $0 \leqq y \leqq 1\}$.

Definition. A continuum $X$ is called a multi-arc in case there exists a continuous, monotone, set-valued function $F$ on the unit interval onto $X$, such that $F(x)$ is a simple arc for each $x$ in the interval. (Here $F(x)$ may be degenerate, i.e., a point.)

Definition. A continuum $X$ is called circularly reducible if and only if there exists a continuous, monotone function $F$ from $X$ onto the circle, such that $F(x)$ is a simple arc for each $x \in X(F(x)$ may be a point).

REMARK. By extending the construction in Example 5, it can be shown that any dendrite with a finite number of branch points is a multi-arc. Note, however, that Example 6 shows that not all multiarcs are locally connected, and that Example 3 shows that the disc is a multi-arc.

From Wallace [14] we have:

Definition. A continuous function $F: X \rightarrow Y$ is anarthric if and only if for each $y \in Y$ no $x \in X-F^{-1}(y)$ separates $F^{-1}(y)$.

Then from the definition of monotone and anarthric we obtain

LEMma 2.2. Let $X$ be a totally ordered, compact, connected space, and let $F: X \rightarrow Y$ be a continuous function on $X$ into $Y$. Then $F$ is anarthric if and only if $F$ is monotone.

Also from [14] we have

THEOREM (Wallace): Let $X$ be compact. A necessary and sufficient condition that a function $F$ on $X$ be anarthric is: If 
$X=M \cup N$, where $M$ and $N$ are continua meeting in a cutpoint $x$, and $K$ is any continuum meeting $M$, then $F(M \cap K)=F(M) \cap F(K)$.

COROLlaRY 2.3. The circle is not a multi-arc.

Proof. Suppose $F:[0,1] \rightarrow C$ is a monotone continuous function on the unit interval onto a circle such that $F(x)$ is an arc for each $x \in[0,1]$. By Lemma $2.2 F$ is anarthric. Thus if $x \in(0,1)$ we have by the theorem $F(x)=F([0, x]) \cap F([x, 1])$. Also $F([0, x]) \cup F([x, 1])=$ $C$ and $F(x)$ is a subarc of $C$. Thus either $F([0, x])$ or $F([x, 1])$ is equal to $C$ for otherwise their intersection would not be connected. Hence we may assume that there exists an $x^{\prime}$ such that $F\left(\left[0, x^{\prime}\right]\right)=$ $F\left(x^{\prime}\right)$ and $F([x, 1])=C$. Let $x_{0}=\sup \left\{x: F([0, x])=F\left(x^{\prime}\right)\right.$ and $F([x$, $1])=C\}$. If $y \in F\left(x_{0}\right)-F\left(x^{\prime}\right)$, and if $U$ is an open set containing $y$ which does not meet $F\left(x^{\prime}\right)$, then $F^{-1}(U)$ is an open set containing $x_{0}$ which does not meet $\left\{x: F([0, x])=F\left(x^{\prime}\right)\right\}$. This contradicts the choice of $x_{0}$. Hence $F\left(x_{0}\right)=F\left(x^{\prime}\right)$ and $F\left(\left[x_{0}, 1\right]\right)=C$. Note $F\left(\left[x_{0}, 1\right]\right)=C$ implies that $x_{0} \neq 1$. Now if $x>x_{0}, F([0, x])=C$ since $x_{0}$ is the sup $\left\{x: F([x, 1])=C\right.$ and $\left.F([0, x])=F\left(x^{\prime}\right)=F\left(x_{0}\right)\right\}$. Thus for $y \in C-F\left(x_{0}\right)$ there is a decreasing sequence $\left\{x_{n}\right\}$ such that $x_{n} \rightarrow x_{0}$ and $y \in F\left(x_{n}\right)$ for all $n$. But this implies that $x_{0} \in F^{-1}(y)$ since $F^{-1}(y)$ is closed, a contradiction.

We can derive more corollaries to Theorem 2.3.

COROLLARY 2.4. A hereditarily unicoherent multi-arc is not circularly reducible.

Proof. Suppose that $x$ is circularly reducible, and that $F_{2}: X \rightarrow C$ is a continuous, monotone function on $X$ onto the circle $C$ such that $F_{2}(x)$ is a simple arc for each $x \in X$. Since $X$ is a multi-arc there exists a continuous, monotone function $F_{1}$ on the unit interval $I$ onto $X$ such that $\mathrm{F}_{1}(r)$ is a simple arc for each $r \in I$. Then by Lemma 2.1 the function $F=F_{2} \circ F_{1}$ is continuous and monotone, and $F$ maps $I$ onto $C$. Now let $M$ be an arc contained in $X$. Then $F_{2} \mid M$ is continuous. Further, if $y \in C$, either $F_{2}^{-1}(y) \cap M=\phi$ or $F_{2}^{-1}(y) \cap M$ is connected since $X$ is hereditarily unicoherent. Therefore $F_{2} \mid M$ is monotone. Hence $F_{2}(M) \neq C$. Further, if $M \in I, F_{1}(r)$ is at most an arc, and hence, $F_{2} \circ F_{1}(r) \neq C$. Note that $F_{2} \circ F_{1}(r)$ is connected. Consequently, $F$ is a continuous, monotone function on $I$ onto $C$ such that $F(r)$ is a simple arc for each $r \in I$; this is a contradiction. Hence the result holds.

COROLlaRY 2.5. A hereditarily unicoherent, arcwise connected continuum is not circularly reducible. 
Proof. We sketch the proof of this result. Let $X$ be an hereditarily unicoherent, arcwise connected continuum. First observe that the set $\{F(x): x \in X\}$ has maximal elements, where $F: X \rightarrow C$ is a monotone function on $X$ onto $C$ such that $F(x)$ is an arc. If $x^{\prime}, x^{\prime \prime}$ are such that $F\left(x^{\prime}\right)$ and $F\left(x^{\prime \prime}\right)$ are maximal, then $F\left(x^{\prime}\right) \cap F\left(x^{\prime \prime}\right) \neq \phi$ and $F\left(x^{\prime}\right) \cup F\left(x^{\prime \prime}\right) \neq C$. From Corollary 2.4, if we have $x_{1}, x_{2}, \cdots, x_{n}$ such that $F\left(x_{1}\right), F\left(x_{2}\right), \cdots, F\left(x_{n}\right)$ are maximal, then $\bigcup_{i=1}^{n} F\left(x_{i}\right) \neq C$. Then the fact that $X$ is compact is used to complete the proof.

3. Nonalternating functions. The purpose of this section is to generalize the definition of nonalternating functions to set-valued functions and to derive some characterizations of such functions.

Definition. A function $F: X \rightarrow Y$ is called nonalternating if and only if for any pair $y_{1}, y_{2} \in F(X)$ there does not exist a separation $X-F^{-1}\left(y_{1}\right)=A \cup B$ such that $y_{2} \in F(A) \cap F(B)$.

Example 7. Let $X=[0,1]$ and define $F: X \rightarrow X$ by $F\left(\frac{1}{2}\right)=\{0\}$, $F(x)=\left[0,2\left(x-\frac{1}{2}\right)\right]$ for $x>\frac{1}{2}$ and $F(x)=\left[0,2\left(\frac{1}{2}-x\right)\right]$ for $x<\frac{1}{2}$. Then $F$ is continuous and nonalternating, but not monotone. Further, this serves as a counterexample to theorems which are true for single-valued functions [18, pp. 138-140].

Definition. A multi-valued function $F: X \rightarrow Y$ is called semisingle-valued (s.s.v.) if and only if $F\left(x_{1}\right) \cap F\left(x_{2}\right) \neq \phi$ implies that $F\left(x_{1}\right)=F\left(x_{2}\right)$.

A very small change will allow us to get the counterpart to Theorem 2.1 [18, p. 138].

THeOREM 3.1. Let $F: X \rightarrow Y$ be continuous. Then $F$ is nonalternating if and only if for each $y \in Y$, and each quasi-component $Q$ of $X-F^{-1}(y), F^{-1}(F(Q)) \cap\left(X-F^{-1}(y)\right)=Q$.

Proof. Suppose that $F$ is nonalternating and that $Q$ is a quasicomponent of $X-F^{-1}(y)$ for $y \in Y$. Then, if

$$
x \in F^{-1}(F(Q)) \cap\left(X-F^{-1}(y)\right)-Q,
$$

there exists a separation $X-F^{-1}(y)=A \cup B$ such that $x \in A$ and $Q \subset B$, as $Q$ is a quasi-component. However, this implies that $F(A) \cap F(B) \neq$ $\phi$, as $x \in F^{-1}(F(Q))$ implies $F(x) \cap F(Q) \neq \phi$ which implies there exists an $x^{\prime} \in Q$ such that $F(x) \cap F\left(x^{\prime}\right) \neq \phi$. This is contrary to the assumption that $F$ is nonalternating. If $F$ is not nonalternating, there exist points $y_{1}, y_{2} \in Y$, and a separation $X-F^{-1}\left(y_{1}\right)=A \cup B$ such that $y_{2} \in$ $F(A) \cap F(B)$. Let $x \in A$ with $y_{2} \in F(x)$ and let $Q$ be the quasi-component 
of $X-F^{-1}\left(y_{1}\right)$ containing $x$. Since $y_{2} \in F(B)$, there exists $x^{\prime} \in B$ such that $y_{2} \in F\left(x^{\prime}\right)$. Hence,

$$
x^{\prime} \in F^{-1}(F(Q)) \cap\left(X-F^{-1}\left(y_{1}\right)\right)-Q,
$$

and the condition fails.

We also obtain

THEOREM 3.2. Let $F: X \rightarrow Y$ be continuous, and let $y \in Y$. Let $Q$ be any quasi-component of $Y-\{y\}$. Then if $F^{-1}(Q) \cap\left(X-F^{-1}(y)\right)$ is contained in a quasi-component of $X-F^{-1}(y), F$ is nonalternating.

Proof. Let $y_{1}, y_{2} \in Y$, and let $Q$ be the quasi-component of $Y-y_{1}$ which contains $y_{2}$. Then, since

$$
F^{-1}\left(y_{2}\right) \cap\left(X-F^{-1}\left(y_{1}\right)\right) \subset F^{-1}(Q) \cap\left(X-F^{-1}\left(y_{1}\right)\right),
$$

the hypothesis implies that for any separation

$$
X-F^{-1}\left(y_{1}\right) A \cup B, A \mid B,
$$

$F^{-1}\left(y_{2}\right) \cap\left(X-F^{-1}\left(y_{1}\right)\right)$ is contained in $A$ or in $B$. Thus, $F$ is nonalternating.

Definitions. Denote the set of all points that separate $a$ and $b$ by $E(a, b)$. Then call $a, b$ conjugate in case $E(a, b)=\phi$. Then, if $x$ is neither a cutpoint nor an end point, the set containing $x$ and all points which are conjugate to $x$ is called a simple link. Finally, a cyclic element of $X$ is either a cutpoint, an end point, or a simple link.

Definition. A connected space is called semi-locally-connected (s.l.c.) at a point $x$ in case $x$ has arbitrarily small neighborhoods whose complements have only a finite number of components. If $X$ is s.l.c. at each of its points, it is called s.l.c.

Using a result of Wallace [14] we can generalize a result on singlevalued functions in [18] to multi-valued functions.

THeOREM (Wallace). A function $F: X \rightarrow Y$ on a continuum $X$ into a continuum $Y$ is anarthric if and only if for any subcontinuum $H$ of $Y$ and any subcontinuum $K$ of $X$ such that $K \cap F^{-1}(H)=$ $P \cup Q, P \mid Q$, there exist points $p \in P, q \in Q$, such that $p$ and $q$ are conjugate.

THEOREM 3.3. Let $F: X \rightarrow Y$ be continuous and semi-single valued, and let $X$ be a semi-locally-connected, metric continuum and $Y$ a 
metric continuum. Then $F$ is nonalternating if and only if the following hold.

(i) $F$ is anarthric,

(ii) $F$ is nonalternating on each cyclic element of $X$.

Proof. Suppose that $F$ is nonalternating. Let $y \in Y$, and suppose there exists a point $p \in E(a, b)-F^{-1}(y)$, where $a, b \in F^{-1}(y)$. Now $y \notin F(p)$, thus $(F(a) \cup F(b)) \cap F(p)=\phi$ since $F$ is semi-single-valued. Moreover, there exists a separation $X-p=A \cup B, A \mid B$, with $a \in A$ and $b \in B$. Let $y^{\prime} \in F(p)$. Then, there exists a separation $A^{\prime}, B^{\prime}$ of $X-F^{-1}\left(y^{\prime}\right)$ such that $a \in A^{\prime}$ and $b \in B^{\prime}$, which implies that $F\left(A^{\prime}\right) \cap$ $F\left(B^{\prime}\right) \neq \phi$. This contradicts the hypothesis that $F$ is nonalternating. Thus, (i) holds.

In order to show (ii) holds, let $E$ be a true cyclic element of $X$ (i.e., a simple link). Let $F(E)=E^{\prime} \subset Y$, and let $y_{1}, y_{2} \in E^{\prime}$. If $E-$ $F^{-1}\left(y_{1}\right) \cap E=A \cup B, A \mid B$ such that $y_{2} \in F(A) \cap F(B)$, then by [18, IV, 3.22 and 6.81], there exists a separation of $X-F^{-1}\left(y_{1}\right)=A^{\prime} \cup B^{\prime}, A^{\prime} \mid B^{\prime}$, with $y_{2} \in F\left(A^{\prime}\right) \cap F\left(B^{\prime}\right)$, a contradiction.

Suppose (i) and (ii) hold. Let $y \in Y$. If $X-F^{-1}(y)=A \cup B$, $A \mid B$, and if $x_{1} \in A, x_{2} \in B$ such that $F\left(x_{1}\right) \cap F\left(x_{2}\right) \neq \phi$, then by the result of Wallace there exist $x_{1}^{\prime}$ and $x_{2}^{\prime}$ which are separated by $F^{-1}(y)$ and which are contained in the same cyclic element, but this contradicts (ii). Thus $F$ is nonalternating.

COROLLARY 3.4. Any nonalternating semi-single-valued fnnction on a dendrite is monotone.

Proof. If $a, b \in F^{-1}(y)$ then by (i), $E(a, b) \subset F^{-1}(y)$, and $E(a, b)$ is a simple arc from $a$ to $b$.

4. Composite functions and factorization. In this section some of the properties of composite functions are investigated and a factorization theorem is obtained.

Definition. A function $F: X \rightarrow Y$ is called open in case whenever $U$ is open in $X, F(U)$ is open in $Y$.

Let $X, Y$ and $Z$ be compact spaces, and let $F, F_{1}$, and $F_{2}$ be continuous functions such that $F_{1}: X \rightarrow Z, F_{2}: Z \rightarrow Y$ and $F=F_{2} \circ F_{1}$, $F: X \rightarrow Y$.

Lemmas 4.1 and 4.2 are extensions of results which hold for single-valued functions. The proofs are straightforward and are omitted.

LEMma 4.1. If $F_{1}$ is single valued: 
(i) $F$ open implies that $F_{2}$ is open;

(ii) $F$ monotone implies that $F_{2}$ is monotone;

(iii) $F$ nonalternating implies that $F_{2}$ is nonalternating.

In addition to this we can obtain:

LEMMA 4.2. The following statements hold.

(i) $F_{1}, F_{2}$ open implies $F$ is open;

(ii) $F_{1}, F_{2}$ monotone implies $F$ is monotone;

(iii) $F_{1}$ monotone and s.s.v., and $F_{2}$ nonalternating imply $F$ is nonalternating.

We now turn to the problem of factoring functions. First we have the known Theorem A, Whyburn [18, pp. 141-142], which is stated below. (Note that Theorem A holds for any compact Hausdorff space, as well as for metric spaces.)

Definition. A function $F: X \rightarrow Y$ is called light in case $F^{-1}(y)$ is totally disconnected for each $y \in Y$.

THEOREM A. Let $g$ be a single-valued, continuous function from $X$ onto $Y$. Then there exist a space $Z$ and continuous functions $g_{1}$, $g_{2} ; g_{1}: X \rightarrow Z, g_{2}: Z \rightarrow Y$, such that $g_{1}$ is monotone, $g_{2}$ is light, and $g=g_{2} \circ g_{1}$.

We can extend this theorem to semi-single-valued functions, but first we need the following lemma.

Lemma 4.3. Let $\mathscr{S} \subset S(X)$, and let $\mathscr{S}$ have the finite topology. Define a function $F: \mathscr{S} \rightarrow X$ by $F(S)=S$ for all $S \in \mathscr{S}$. Then $F$ is continuous.

Proof. Let $U$ be an open set contained in $X$. If $S \in \mathscr{S}$ and $S \cap U \neq \phi$, the set $\langle U, X\rangle=\{S \in \mathscr{S}: S \cap U \neq \phi\}$ is an open set in $\mathscr{S}$ such that $F(S) \cap U \neq \phi$ for all $S \in\langle U, X\rangle$. If $S \subset U$, then $\langle U\rangle=$ $\{S \in \mathscr{S}: S \subset U\}$ is an open set in $\mathscr{S}$ such that $F(\langle U\rangle) \subset U$. Thus, by Lemma $1.1, F$ is continuous.

Note. If $\mathscr{S}$ is a decomposition, then $F^{-1}(x)=\{S\}$ where $x \in S$, and if $F: X \rightarrow Y$ is semi-single-valued, then $\mathscr{S}=\{F(x): x \in X\}$ is a decomposition.

THEOREM 4.5. Let $F: X \rightarrow Y$ be continuous and semi-single-valued. Then there exist a space $Z$ and continuous functions $F_{1}, F_{2}$ with $F_{1}: X \rightarrow Z$ single-valued, $F_{2}: Z \rightarrow Y, F=F_{2} \circ F_{1}$, and such that $F_{1}$ is monotone, and $F_{2}$ is light. 
Proof. Let $f$ be the induced single-valued function on $X$ into $S(Y)$. Then $f(X)=\{F(x): x \in X\}$ is a decomposition of $Y$. Then by Theorem A there exist a space $Z$ and continuous functions $f_{1}, f_{2}$ such that $f_{1}$ is monotone, $f_{2}$ is light, and $f=f_{2} \circ f_{1}$. Let $F^{*}$ be the function of Lemma 4.3. Then set $F_{1}=f_{1}$ and $F_{2}=F^{*} \circ f_{2}$. Thus, $F_{1}$ is single valued and monotone and, from the remark following Lemma 4.3, $F_{2}$ is continuous and light. Finally, $F=F_{2} \circ F_{1}$.

Finally, with Lemma 4.1 (i), we get:

CoRollary 4.6. If $F: X \rightarrow Y$ is semi-single-valued, continuous and open, then there exist continuous functions $F_{1}, F_{2}$ such that $F_{1}$ is single-valued and monotone, and $F_{2}$ is light and open, and such that $F=F_{2} \circ F_{1}$.

5. Semi-single-valued functions, Let $F: X \rightarrow Y$ be a semisingle-valued continuous function from $X$ onto $Y$, and define the collections $Q=\{F(x): x \in X\}$, and $P=\left\{F^{-1}(y): y \in Y\right\}$. That $P$ and $Q$ are decompositions into disjoint closed sets follows from the definition of a continuous, semi-single-valued function.

Let $q: Y \rightarrow Q$ and $p: X \rightarrow P$ be the projections of $Y$ onto $Q$ and $X$ onto $P$, respectively. Define $F^{\sharp}$ on $P$ onto $Y$ by $F^{\sharp}(D)=F(x)$ for $D \in P$ and $x \in D$, and define $f^{\prime}$ on $X$ onto $Q$ by $f^{\prime}(x)=F(x)$. Note that $f^{\prime}$ and $f$ are essentially the same but $Q$ as a decomposition has the quotient topology rather than the finite topology. When $F$ is the inverse of a single-valued function, we have by Theorem 5.10 [6], that the quotient and the finite topologies are equivalent. We shall generalize this result in Corollary 5.3. Finally, define $f^{*}: P \rightarrow Q$ by $f^{*}(D)=F(x)$ for $D \in P$ and $x \in D$.

THEOREM 5.1. If $X$ and $Y$ are compact, the decompositions $P$ and $Q$ are upper semi-continuous. Further, $P$ and $Q$ are Hausdorff in the quotient topology.

Proof. Let $V_{1}, V_{2}$ be disjoint open subsets of $Y$ such that $F\left(x_{1}\right) \subset$ $V_{1}$ and $F\left(x_{2}\right) \subset V_{2}$. Then, for $i=1,2, Y-F F^{-1}\left(Y-V_{i}\right)$ is an open set containing $F\left(x_{i}\right)$ which is contained in $V_{i}$ and which is the union of members of $Q$. Similarly, if $F^{-1}\left(y_{1}\right) \neq F^{-1}\left(y_{2}\right)$ are in $P$ and if $U_{1}$ and $U_{2}$ are open and disjoint with $F^{-1}\left(y_{i}\right) \subset U_{i}$, then $X-F^{-1} F\left(X-U_{i}\right)$, $i=1,2$, are the required open sets. This shows that $P$ and $Q$ are upper semi-continuous, and Hausdorff in the quotient topology.

THEOREM 5.2. The functions $F^{*}$ and $f^{\prime}$ are continuous when $P$ and $Q$ have the quotient topology. 
Proof. Since $F^{\sharp}(D)=F\left(p^{-1}(D)\right)$ for $D \in P$, Theorem 5.1 implies that $F^{\#}$ is continuous. Also $f^{\prime}=q \circ F$ and hence is continuous by Lemma 1.12.

Now we obtain a generalization of Theorem 5.10 [6].

CoRollary 5.3. If $X$ and $Y$ are compact and $F: X \rightarrow Y$ is a semi-single-valued continuous function, then the finite and quotient topologies agree on $Q=\{F(x): x \in X\}$, and $f$ and $f^{\prime}$ are equivalent functions.

Proof. The function $F^{\#}$ is the inverse of a single-valued function. Hence, Theorem 5.10 [6] applies.

THEOREM 5.4. The function $f^{*}: P \rightarrow Q$ is a homeomorphism onto, when $X$ and $Y$ are compact.

Proof. That $f^{*}$ is a single-valued function which is 1 to 1 and onto follows immediately from the fact that $F$ is semi-single-valued. That $f^{*}$ is continuous follows from $f^{*}=q \circ F^{*}$, Theorem 5.2 and Corollary 5.3.

We associate with each multi-valued function $F: X \rightarrow Y$ the induced function $f$ on $X$ into $S(Y)$ and we can define a function $F^{*}$ on $f(X)$ into $Y$ by $F^{*}(f(x))=F(x)$. Then $F=F^{*} \circ f$. We consider briefly the relationships between $F, f$ and $F^{*}$ and the properties of being monotone, open, and nonalternating. A typical question is: "Does $F$ monotone imply that $f$ is monotone, and conversely?" Simple examples show that $f$ monotone does not imply that $F$ is monotone, and Example 8 shows that the converse fails.

EXAMPLE 8 . Let $X$ be the rectangle with corners $(0,-1),(1$, $-1),(1,1)$ and $(0,1)$ together with its interior. Let $Y$ be the unit interval. Let $(x, y) \in X$ and define $r_{1}=\frac{1}{2}(1-x), r_{2}=\frac{1}{2}(1+x)$. Then define $z_{1}=r_{1}(1-|y|), z_{2}=r_{1}+|y|\left(\frac{1}{2}-r_{1}\right), z_{3}=r_{2}-|y|\left(r_{2}-\frac{1}{2}\right)$ and $z_{4}=r_{2}+|y|\left(1-r_{2}\right)$ with $r_{i}, z_{j} \in Y$. Define $F: X \rightarrow Y$ by $F((x, y))=$ $\left[z_{1}, z_{2}\right] \cup\left[z_{3}, z_{4}\right]$. Then $F$ is monotone and continuous but $f$ is not monotone.

However, if $F$ is semi-single-valued, we have

THEOREM 5.5. If $F: X \rightarrow Y$ is a semi-single-valued, continuous function from $X$ onto $Y$, then $F$ is monotone if and only if $f$ is monotone.

Proof. If $y \in Y$, then there exists a unique $S$ in $F(X)$ such that 
$y \in S$. Thus $F^{-1}(y)=\{x: F(x)=S\}=f^{-1}(S)$. So $F^{-1}(y)$ is connected if and only if $f^{-1}(S)$ is connected.

THEOREM 5.6. The following statements hold.

(i) $F$ monotone implies $F^{*}$ is monotone.

(ii) $F$ open implies $F^{*}$ is open.

(iii) If $F$ is semi-single-valued, $F$ open implies $f$ is open.

(iv) $F$ nonalternating implies $F^{*}$ is nonalternating.

Further, we may state a partial converse to (i), (ii) and (iv).

THEOREM 5.7. If $f$ is monotone, then

(i) $F^{*}$ monotone implies that $F$ is monotone; and

(ii) $F^{*}$ nonalternating implies that $F$ is nonalternating.

THEOREM 5.8. If $f$ is open, $F^{*}$ open implies $F$ is open.

6. Open functions, The purpose of this section is to show that certain results in Whyburn [18] on open mappings can be generalized to semi-single-valued functions and in some cases to arbitrary multivalued functions. In this section all spaces will be separable, metric spaces.

REMARK 1. The definition of terms used in this section are those of Whyburn [18].

Remark 2. If $X$ is compact, then a collection of subsets $G$ of $X$ is continuous if and only if it is continuous in the limit sense.

THEOREM 6.1. Let $F: X \rightarrow Y$ be a continuous, semi-single-valued function of $X$ onto $Y$. If $F$ is open, then the collection $\left\{F^{-1}(y)\right.$ : $y \in Y\}$ is continuous in the limit sense. Conversely, if $X$ is compact, and if the collection $\left\{F^{-1}(y): y \in Y\right\}$ is continuous, then $F$ is open.

Proof. By Theorem 5.6, $F$ open implies $f$ open and since $F$ is s.s.v., $f^{-1}(F(x))=F^{-1}(y), y \in F(x)$. Thus, the first statement follows from the theorem for single-valued functions [18, Theorem 4.31, p. 130], and minor modifications of the proof in [18] will yield a proof of the converse.

COROLlary 6.2. Let $X$ be compact and let $F$ be as in Theorem 6.1. Then $F$ is open if and only if the collection $\left\{F^{-1}(y): y \in Y\right\}$ is continuous.

We can also generalize a theorem due to Eilenberg, [18, p. 138]. 
THEOREM 6.3. Let $F: X \rightarrow Y$ be continuous, semi-single-valued, and onto. Then $F$ is open if and only if for each sequence $\left\{y_{n}\right.$ : $n=1, \cdots\}$ in $Y$ such that $\lim y_{n}=y_{0}, \lim F^{-1}\left(y_{n}\right)=F^{-1}\left(y_{0}\right)$.

Proof. Suppose that $F$ is open, and that $\left\{y_{n}\right\}$ is a sequence in $Y$ such that $\lim y_{n}=y_{0}$. In view of Theorem 6.1 we need only show that $F^{-1}\left(y_{0}\right)^{n} \cap \lim \inf F^{-1}\left(y_{n}\right) \neq \phi$. If $x \in F^{-1}\left(y_{0}\right)$, if $U$ is an open set containing $x$, and if $U \cap F^{-1}\left(y_{n}\right)=\phi$ for infinitely many $n$, then $F(U)$ is an open set containing $y_{0}$ such that $y_{n} \notin F(U)$ for infinitely many $n$, a contradiction to $\lim _{n} y_{n}=y_{0}$.

Now suppose that $\lim y_{n}=y_{0}$ implies $\lim F^{-1}\left(y_{n}\right)=F^{-1}\left(y_{0}\right)$, and let $U$ be open in $X$. If $F(U)$ is not open in $Y$, there exists $y_{0} \in U$ and a sequence $\left\{y_{n}\right\} \subset Y-F(U)$ such that $\lim y_{n}=y_{0}$. Now $y_{0} \in F(U)$ implies that there exists an $x \in F^{-1}\left(y_{0}\right) \cap U$, and from the hypothesis. $U \cap F^{-1}\left(y_{n}\right) \neq \phi$ for all but finitely many $n$. Thus $y_{n} \in F(U)$ for all but finitely many $n$, a contradiction. Hence $F$ is open.

The proof of the following lemma is straightforward and is omitted. Note that in many of the following results the restriction to separable metric spaces is unnecessary.

Lemma 6.4. Let $F: X \rightarrow Y$ be continuous and onto. Then $Q \subset X$ is an inverse set if and only if $F(A \cap Q)=F(A) \cap F(Q)$ for each $A \subset X$.

Lemma 6.5. Let $F: X \rightarrow Y$ be continuous and open. If $Q \subset X$ is an inverse set, then $F$ restricted to $Q$ is open.

Proof. Let $V$ be open in $Q$. Then there exists an open set $U$ in $X$ such that $V=Q \cap U$. Then, by Lemma 6.4, $F(V)=F(U \cap Q)=$ $F(U) \cap F(Q)$, which is open in $F(Q)$ since $F(U)$ is open.

In order to establish the next result we need a theorem of Michael's [6, Theorem 2.5.1].

TheOREM B. If $X$ is regular, and $B \subset S(X)$ is compact, then $\cup\{E: E \in B\}$ is closed.

THEOREM 6.6. Let $F: X \rightarrow Y$ be onto and continuous. Then, if $A \subset X$ is conditionally compact:

(i) $\overline{F(A)}=F(\bar{A})$;

(ii) $\overline{F(A)}-F(A) \subset F(\bar{A}-A)$.

Further, if $F$ is an open function, and $A$ is an open set,

(iii) $b(F(A)) \subset F(b(A))$

where $b(A)$ denotes the boundary of $A$. 


\section{Proof.}

(i) Let $A \subset X$ be conditionally compact. Then, by Theorem $\mathrm{B}$, $F(\bar{A})$ is closed. Hence $\overline{F(A)} \subset F(\bar{A})$. Also $F(\bar{A}) \subset \overline{F(A)}$ since $F$ is continuous.

(ii) From (i), $\overline{F(A)}-F(A)=F(\bar{A})-F(A) \subset F(\bar{A}-A)$.

(iii) With $A$ open and $F$ open this is immediate from (ii).

LEMMA 6.7. Let $U, U_{1}, U_{2}$ be open sets such that $U=U_{1} \cup U_{2}$. If $U_{1} \cap U_{2}=\phi$, then $b(U)=b\left(U_{1}\right) \cup b\left(U_{2}\right)$.

Proof. If $x \in b(U)$, then $x \in \bar{U}_{1}$ or $x \in \bar{U}_{2}$ and $x \notin U_{1} \cup U_{2}$. Therefore $x \in b\left(U_{1}\right)$ or $x \in b\left(U_{2}\right)$. On the other hand $x \in b\left(U_{i}\right)$ implies $x \in \bar{U}_{i}$ and $x \notin U_{1} \cup U_{2}$. Thus $x \in b(U)$.

Theorem 6.8. Let $X$ and $Y$ be continua, and let $F: X \rightarrow Y$ be continuous, open and onto. Then:

(i) If $X$ is a curve of order less than or equal to $n$, and if $F(x)$ contains at most $m$ points for each $x \in X$, then $Y$ is a curve of order less than or equal to $\mathrm{nm}$;

(ii) If $X$ is a regular curve and if $F(x)$ is finite for each $x$, then $Y$ is a regular curve; and

(iii) If $X$ is a rational curve and $F(x)$ countable for each $x$, then $Y$ is a rational curve.

\section{Proof.}

(i) Let $y \in Y$, and let $V$ be an open set containing $y$. Since $F$ is onto, there exists an $x \in X$ such that $y \in F(x)$. Let $F(x)=$ $\left\{y_{1}, \cdots, y_{k}\right\}, k \leqq m$. Suppose $y=y_{1}$. Then there exist open sets $V, V^{\prime}$ of $Y$ such that $y_{1} \in V,\left\{y_{2}, \cdots, y_{k}\right\} \subset V^{\prime}$ and $V \cap V^{\prime}=\phi$. Further there exists an open set $U$ containing $x$ such that $F(U) \subset V \cup V^{\prime}$ and such that $b(U)$ contains at most $n$ points (as $X$ is a curve of order less than or equal to $n)$. Let $V_{1}=F(U) \cap V$ and $V_{2}=F(U) \cap V^{\prime}, V_{1}$ and $V_{2}$ are open and disjoint. Thus, by Lemma 6.7, $b(F(U)) \subset b\left(V_{1}\right) \cup b\left(V_{2}\right)$. Therefore by Theorem 6.6, $b\left(V_{1}\right) \subset b(F(U)) \subset F(b(U))$ and this latter set contains at most $n m$ points. Thus $V_{1}$ is the required open set containing $y$.

(ii) A proof similar to the proof of (i) will establish (ii).

(iii) Let $x \in Y$, and let $V$ be an open set containing $y$. Pick an $x \in X$ such that $y \in F(x)$. Since $F(x)$ is countable, we may assume that $F(x) \subset V \cup(Y-\bar{V})$. Since $X$ is rational, there exists an open set $U$ containing $X$ such that $F(U) \subset V \cup(Y-\bar{V})$, with $b(U)$ countable. By part (iii) of Theorem 6.6, $b(F(U)) \subset F(b(U))$ and $F(b(U))$ is countable. Then $F(U) \cap V$ is an open set containing $y$ 
with countable boundary. This last since

$$
b(F(U))=b(V \cap F(U)) \cup[b(Y-\bar{V}) \cap F(U)],
$$

by Lemma 6.7 .

Following Whyburn's proof $[18$, p. $147,7.4]$, we can prove

THeOREM 6.9. Let $X$ be compact and let $F: X \rightarrow Y$ be continuous, open and onto. If $A$ is a connected open set in $Y$, and if $Q$ is any quasi-component of $F^{-1}(A)$, then $A \subset F(Q)$.

Corollary 6.10. Let $X$ and $Y$ be locally connected, compact spaces, $F: X \rightarrow Y$ open and onto, and let $A$ be any closed set in $Y$. If $C$ is any component of $Y-A$, then $F^{-1}(C)$ has only a finite number of components and each of these maps onto all of $C$ under $F$.

Proof. It follows from the hypothesis that any quasi-component of $F^{-1}(C)$ is also a component of $F^{-1}(C)$. Then if $F^{-1}(C)$ has an infinite number of quasi-components, a sequence constructed by choosing one element from each quasi-component must have a limit point. However, each quasi-component is open; hence no subsequence can converge to the limit point, a contradiction. Finally, $C$ is open so Theorem 6.5 implies that $C \subset F(Q)$ for any quasi-component $Q \subset F^{-1}(C)$.

Proposition 6.11. Let $F: X \rightarrow Y$ be open and onto, and let $Y$ be connected. If $X_{0}$ is an inverse set in $X$ which is open and closed, then $F\left(X_{0}\right)=Y$.

Proof. Since $X_{0}$ is an inverse set and $F$ is open, $F\left(X_{0}\right)$ and $F\left(X-X_{0}\right)$ are disjoint open sets whose union is $Y$. Therefore, $F\left(X_{0}\right)=Y$.

Remark. Let $F: X \rightarrow Y$ be continuous. If $C$ is a subset of $Y$, then $F^{-1}(C)$ need not be an inverse set. However, if $F$ is an s.s.v. function, we have:

Lemma 6.12. Let $F: X \rightarrow Y$ be an s.s.v. function. If $C \subset Y$, then $F^{-1}(C)$ is an inverse set.

Proof. If $x \in F^{-1} F F^{-1}(C)$, then $F(x) \cap F\left(F^{-1}(C)\right) \neq \phi$. Thus there exists an $x^{\prime} \in F^{-1}(C)$ such that $F(x) \cap F\left(x^{\prime}\right) \neq \phi$. Then since $F$ is s.s.v., $F(x)=F\left(x^{\prime}\right)$. Therefore, $F(x) \cap C \neq \phi$, and hence $x \in F^{-1}(C)$. Consequently, $F^{-1}(C)$ is an inverse set.

THEOREM 6.13. Let $X$ be compact, and let $F: X \rightarrow Y$ be a con- 
tinuous, open semi-single-valued function on $X$ onto $Y$. Let $C$ be any compact, connected set in $Y$. Then for any component $K$ of $Q=$ $F^{-1}(C)$, it follows that $C \subset F(K)$.

Proof. Since $F$ is s.s.v., $F^{-1}(Q)$ is an inverse set in $X$ by Lemma 6.12. Hence, by Lemma 6.5, $F$ restricted to $Q$ is open and the result follows by applying Theorem 6.9 to $F$ restricted to $Q$.

Note. Single-valued open, continuous functions map nodal sets onto nodal sets ( $A$ is nodal in case $A \cap \overline{X-A}$ is at most a single point), but Example 3 is a counterexample to this result for s.s.v. mappings. In fact, in Example $3, F$ is the inverse of a continuous single-valued function.

7. Quasi-monotone functions, In this section $X$ and $Y$ are compact and connected, and $F: X \rightarrow Y$ will always denote a continuous function of $X$ onto $Y$.

Definition. A function $F$ is called quasi-monotone in case for each continuum $Y_{0} \subset Y$ with nonvoid interior, $F^{-1}\left(Y_{0}\right)$ has only a finite number of components $C_{n}$ and $Y_{0} \subset F\left(C_{n}\right)$ for each component $C_{n}$ of $F^{-1}\left(Y_{0}\right)$. Note that any monotone function on a continuum is quasimonotone.

REMARK. If $g$ is a continuous single-valued function on a compact, connected, locally connected space $X$, then $g(X)$ is also compact, connected and locally connected. However, when $F$ is multi-valued this may not be the case, so it is sometimes necessary to assume that $Y$ as well as $X$ is compact, connected, and/or locally connected.

The proof of Theorem 7.1 is very much like the proof of the corresponding theorem for single-valued functions [18, p. 152, Th. 8.1] and is omitted.

THEOREM 7.1. Let $X$ and $Y$ be locally connected continua, and let $F: X \rightarrow Y$. Then $F$ is quasi-monotone if and only if for each component $C$ of the inverse of any connected open set $V$ of $Y, V \subset$ $F(C)$.

COROLlARY 7.2. Every open function on a locally connected continuum onto a locally connected continuum is quasi-monotone.

Proof. Corollary 6.10 implies that the hypotheses of Theorem 7.1 are satisfied. 
THEOREM 7.3. If $X$ and $Y$ are locally connected continua, and if $F$ is light, then $F$ is quasi-monotone if and only if $F$ is open.

Proof. If $F$ is open, then $F$ is quasi-monotone by Corollary 7.2. Suppose that $F$ is quasi-monotone, let $U$ be open in $X$, and let $y \in$ $F(U)$. If $x \in U \cap F^{-1}(y)$, then since $F$ is light there exists a connected open set $U^{\prime} \subset U$ such that $x \in U^{\prime}$ and $b\left(U^{\prime}\right) \cap F^{-1}(y)=\phi$. Let $Q$ be the component of $Y-F\left(b\left(U^{\prime}\right)\right)$ containing $y$, and let $C$ be the component of $F^{-1}(Q)$ containing $x$. Then $C \subset U^{\prime}$ since $C \cap b\left(U^{\prime}\right)=\phi$, and by Theorem 7.1, $Q \subset F(C)$. Then $Q \subset F(C) \subset F\left(U^{\prime}\right) \subset F(U)$ and $Q$ is an open set containing $y$. Thus $F(U)$ is open.

THeOREM 7.4. Let $X, Y$ and $Z$ be locally connected, and let $F=F_{2} \circ F_{1}, F_{1}: X \rightarrow Z, F_{2}: Z \rightarrow Y$ with $F_{1}$ and $F_{2}$ continuous and onto. Then:

(i) If $F$ is quasi-monotone and $F_{1}$ is single-valued, $F_{2}$ is quasimonotone; and

(ii) If $F_{1}$ and $F_{2}$ are quasi-monotone, $F$ is quasi-monotone.

Proof.

(i) Let $V$ be an open connected set in $Y$, and let $C$ be a component of $F_{2}^{-1}(V)$. Let $C^{\prime}$ be a component of $F^{-1}(V)$ contained in $F_{1}^{-1}(C)$. Then, since $F_{1}$ is single-valued, $F_{1}\left(C^{\prime}\right) \subset C$, and since $F$ is quasi-monotone, $V \subset F_{2} \circ F_{1}\left(C^{\prime}\right)=F(C)$. Therefore, $V \subset F_{2}(C)$, as $F_{2} \circ F_{1}\left(C^{\prime}\right) \subset F_{2}(C)$, and $F_{2}$ is quasi-monotone by Theorem 7.1.

(ii) Let $V$ be an open connected set in $Y$. Let $C$ be a component of $F^{-1}(V)$, and let $Q$ be a component of $F_{2}^{-1}(V)$ such that $C$ contains a component of $F_{1}^{-1}(Q)$. Then, since $F_{1}$ is quasi-monotone, $Q \subset F_{1}(C)$. Further, $\quad F_{2}$ quasi-monotone implies that $V \subset F_{2}(Q)$. Thus $V \subset$ $F_{2} \circ F_{1}(C)=F(C)$.

THeOREM 7.5. Let $X$ and $Y$ be locally connected and let $F: X \rightarrow Y$ be s.s.v. Then $F$ is quasi-monotone if and only if there exists a locally connected continuum $Z$, a continuous monotone function $F_{1}$ of $X$ onto $Z$ and a continuous, light, open function $F_{2}$ of $Z$ onto $Y$ such that $F=F_{2} \circ F_{1}$.

Proof. If such a $Z, F_{1}$, and $F_{2}$ exist, then $F$ is quasi-monotone by Corollary 7.2 and by Theorem 7.4, Part (ii). If $F$ is quasi-monotone, then by Theorem 4.5 there exists a continuum $Z$, and a monotone, single-valued function $F_{1}$ of $X$ onto $Z$, and there exists a continuous, light function $F_{2}$ of $Z$ onto $Y$, such that $F=F_{2} \circ F_{1}$. By Theorem 7.4, $F_{2}$ is quasi-monotone and therefore, by Theorem $7.3, F_{2}$ is open. 
Finally, combining the results of Theorem 7.3, the fact that monotone functions are quasi-monotone, and Theorem 7.5, we have the following result for semi-single-valued functions.

THEOREM 7.6. A topological property of locally connected continua is invariant under quasi-monotone, semi-single-valued functions if and only if it is invariant under both monotone and light, open, semi-single-valued functions.

8. Local properties and functions with finite images. In previous sections we have exhibited examples of functions that did not preserve local properties. We saw that even if $F(x)$ was an arc for each $x$, the image of the unit interval need not be locally connected. The purpose of this section is to show that if $F(x)$ is finite for each $x \in X$, then local properties may be preserved. The main theorem is this: If $F$ is defined on a locally connected metric continuum $X$ onto a metric continuum $Y$, and if $F(x)$ is finite for each $x$, then $Y$ is locally connected.

Notation. Designate the number of points in $F(x)$ by $N(F(x))$, and if $N(F(x)) \leqq n$ for all $x$, then write $N(F) \leqq n . \quad N(F)=n$ means that $N(F) \leqq n$ and there is at least one $x$ such that $N(F(x))=n$. If $F(x)$ is finite for each $x$, write $N(F)<\infty$. Finally, $N(F) \equiv n$ means $N(F(x))=n$ for all $x \in X$.

Lemma 8.1. Let $F: X \rightarrow Y$ be continuous with $N(F)<\infty$. If $K$ is a connected subset of $X$, then $F(K)$ has at most $n$ components, where $n=\min N(F(x))$. If $C$ is a component of $F(K)$ and if $x \in K$, then $F(x) \cap C \neq \phi$.

Proof. Let $C$ be a component of $F(K)$ and let $x \in K$. Suppose $F(x) \cap C=\phi$. Define $K_{1}=\{x \in K: F(x) \cap C=\phi\}$ and $k_{2}=\{x \in K: F(x) \cap$ $C \neq \phi\}$. Clearly $K_{1}, K_{2} \neq \phi$ and $K=K_{1} \cup K_{2}$. Also $K_{2} \subset F^{-1}(\bar{C})$ and so $\bar{K}_{2} \cap K_{1}=\phi$. If $x \in \bar{K}_{1} \cap K_{2}$, then $F(x) \cap C \neq \phi$ and $x \in \bar{K}_{1}$ implies there is an $x^{\prime} \in K_{1}$ such that $F\left(x^{\prime}\right) \cap C \neq \phi$, a contradiction. Hence $F(x) \cap C \neq \phi$. Finally since $n=\min N(F(x)), x \in K$, there can be at most $n$ components of $F(K)$.

Proposition 8.2. Let $F: X \rightarrow Y$ be open, continuous, and onto with $N(F)<\infty$. Then the following statements hold:

(i) $X$ locally compact implies $Y$ locally compact;

(ii) $X$ locally connected implies $Y$ locally connected.

Proof. Both proofs are done at once. Let $y \in Y$ and $x \in X$ such 
that $y \in F(x)$. Let $F(x)=\left\{y, y_{1}, \cdots, y_{k}\right\}$, and let $V_{0}, V_{1}, \cdots, V_{k}$ be disjoint open sets containing $y, y_{1}, \cdots, y_{k}$, respectively. Then there exists an open set $U$ with $\bar{U}$ compact (or $U$ connected) such that $F(\bar{U}) \subset \bigcup_{i=0}^{k} V_{i}$, and $F(\bar{U}) \cap V_{j} \neq \phi$ for all $j$. Then since $F$ is open, $F(U)$ and hence $F(U) \cap V_{0}$ is open. Further, $F(\bar{U}) \subset \bigcup_{i=0}^{k} V_{i}$. Thus, when $\bar{U}$ is compact, $F(\bar{U}) \cap V_{0}$ is compact and (i) is proved. Moreover, when $U$ is connected, $F(U)$ has exactly $k+1$ components $C_{i}$, each of which is open. If $C_{0}$ is the component of $F(U)$ containing $y$, then $C_{0} \subset V_{0}$ and $C_{0}$ is connected. Hence $Y$ is locally connected.

We now state one of the main results of this section.

THeOREM 8.3. Let $X$ and $Y$ be compact metric spaces and let $F: X \rightarrow Y$ be continuous and onto with $N(F)<\infty$. If $X$ is locally connected, then $Y$ is locally connected.

Proof. We shall show that $Y$ has property $S$. Let $\varepsilon>0$. Let $x \in X$ and $F(x)=\left\{y_{1}, \cdots, y_{k}\right\}$. There exist open sets $V_{1}, \cdots, V_{k}$ in $Y$ with $d\left(V_{i}\right)<\varepsilon$ for each $i$, and $V_{i} \cap V_{j}=\phi, i \neq j$, such that $y_{i} \in V_{i}$ for each $i$. Since $X$ is compact and locally connected, there exists an open connected set $U_{x}$ containing $x$ such that $F\left(U_{x}\right) \subset \bigcup_{i=1}^{k} V_{i}$. Thus, by Lemma 8.1, $F\left(U_{x}\right)$ has $k$ components each of which has diameter less than $\varepsilon$. We obtain such a $U_{x}$ for each $x$ and extract a finite subcover $U_{x_{1}}, \cdots, U_{x_{q}}$. Then, if $F\left(U_{x_{j}}\right)$ has components $C_{j_{1}}, \cdots, C_{j_{j}}$, the collection $\left\{C_{i j}: i=1, \cdots, q, j=1, \cdots, n_{i}\right\}$ is a finite cover of $Y$ by connected sets of diameter less than $\varepsilon$. Hence, $Y$ has property $S$ and is locally connected.

CoROLLARY 8.4. Let $X$ be a locally connected, metric continuum, $Y$ a metric space, and let $F: X \rightarrow Y$ be continuous. If $N(F)<\infty$, and $\min N(F(x))=n$, then $F(X)$ is the union of at most $n$ locally connected, metric continua.

Proposition 8.5. If $F: X \rightarrow Y$ is a continuous function with $N(F) \leqq n$ and if $C$ is a component of $F(X)$, then $F^{*}: X \rightarrow C$ defined by $F^{*}(x)=F(x) \cap C$ is continuous with $N\left(F^{*}\right) \leqq n-r$, where $r$ is the number of other components of $F(X)$.

Proof. Since $C$ is a component of $F(X)$ there is an open subset of $Y$ which contains $C$ and does not meet any other component of $F(X)$. By Lemma 8.1, $F(x) \cap C \neq \phi$ for all $x \in X$. Thus the result follows by Lemma 1.1.

9. Acknowledgements. This paper is based on the author's doctoral dissertation presented to the University of Oregon. The 
author wishes to express his deep appreciation to Professor L. E. Ward, Jr. for his invaluable aid during the preparation of this paper.

The author would also like to thank the referee for a valuable suggestion which allowed one of the proofs to be shortened considerably.

\section{REFERENCES}

1. C. Berge, Espaces topologiques: fonctions multivoque, Collections Universitaire de Mathematique, III. Dunod, Paris, 1959.

2. G. Choquet, Convergences, Grenoble Université Annales; 23, (1947), 57-112.

3. S. Eilenberg and D. Montgomery, Fixed point theorems for multi-valued transformations, Amer. J. Math. 68 (1946), 214-222.

4. O. Frink, Jr., Topology in lattices, Trans. Amer. Math. Soc. 51 (1942), 569-582.

5. J. L. Kelley, General topology, Princeton, 1955.

6. E. Michael, Topologies on spaces of subsets, Trans. Amer. Math. Soc. 71 (1951), $152-182$.

7. R. L. Plunkett, A fixed point theorem for continuous multi-valued transformations, Proc. Amer. Math. Soc. 7 (1956), 160-163.

8. R. H. Rosen, Fixed points for multi-valued functions on snake-like continua, Proc. Amer. Math. Soc. 10 (1959), 167-173.

9. W.S trother, On an open question concerning fixed points, Proc. Amer. Math. Soc. 4 (1953), 988-993.

10. — Multi-homotopy, Duke J. 22 (1955), 281-285.

11. — Fixed points, fixed sets, and M-retracts, Duke J. 22 (1955), 551-556.

12. W. Strother, Continuous multi-valued functions, Boletim da Sociedade de S. Paulo 10 (1958), 87-120.

13. A. D. Wallace, $A$ fixed point theorem for trees, Bull. Amer. Math. Soc. 47 (1941), 757-760.

14. _ Cyclic invariance under multi-valued maps, Bull. Amer. Math. Soc. 55 (1949), 820-824.

15. L. E. Ward, Jr., A fixed point theorem for multi-valued functions, Pacific J. Math. 8 (1958), 921-927.

16. _ - A fixed point theorem for chained spaces, Pacific J. Math. 9 (1959), 12731278.

17. - Characterization of the fixed point property for a class of set valued mappings, Fundamenta Mathematicae, L(1961), 159-164.

18. G. T. Whyburn, Analytic topology, AMS Colloquium, 28 (1942). 



\title{
PACIFIC JOURNAL OF MATHEMATICS
}

\author{
EDITORS
}

\author{
H. Samelson \\ Stanford University \\ Stanford, California \\ R. M. Blumenthal \\ University of Washington \\ Seattle, Washington 98105
}

\author{
J. DugundjI \\ University of Southern California \\ Los Angeles, California 90007 \\ Richard Arens \\ University of California \\ Los Angeles, California 90024
}

\section{ASSOCIATE EDITORS}
E. F. BECKENBACH
B. H. NEUMaNN
F. WOLF
K. YosIDA

\section{SUPPORTING INSTITUTIONS}

\author{
UNIVERSITY OF BRITISH COLUMBIA \\ CALIFORNIA INSTITUTE OF TECHNOLOGY \\ UNIVERSITY OF CALIFORNIA \\ MONTANA STATE UNIVERSITY \\ UNIVERSITY OF NEVADA \\ NEW MEXICO STATE UNIVERSITY \\ OREGON STATE UNIVERSITY \\ UNIVERSITY OF OREGON \\ OSAKA UNIVERSITY \\ UNIVERSITY OF SOUTHERN CALIFORNIA
}

\author{
STANFORD UNIVERSITY \\ UNIVERSITY OF TOKYO \\ UNIVERSITY OF UTAH \\ WASHINGTON STATE UNIVERSITY \\ UNIVERSITY OF WASHINGTON \\ AMERICAN MATHEMATICAL SOCIETY \\ CALIFORNIA RESEARCH CORPORATION \\ SPACE TECHNOLOGY LABORATORIES \\ NAVAL ORDNANCE TEST STATION
}

Mathematical papers intended for publication in the Pacific Journal of Mathematics should by typewritten (double spaced). The first paragraph or two must be capable of being used separately as a synopsis of the entire paper. It should not contain references to the bibliography. Manuscripts may be sent to any one of the four editors. All other communications to the editors should be addressed to the managing editor, Richard Arens, at the University of California, Los Angeles, California 90024.

50 reprints per author of each article are furnished free of charge; additional copies may be obtained at cost in multiples of 50 .

The Pacific Journal of Mathematics is published quarterly, in March, June, September, and December. Effective with Volume 13 the price per volume (4 numbers) is $\$ 18.00$; single issues, $\$ 5.00$. Special price for current issues to individual faculty members of supporting institutions and to individual members of the American Mathematical Society: $\$ 8.00$ per volume; single issues $\$ 2.50$. Back numbers are available.

Subscriptions, orders for back numbers, and changes of address should be sent to Pacific Journal of Mathematics, 103 Highland Boulevard, Berkeley 8, California.

Printed at Kokusai Bunken Insatsusha (International Academic Printing Co., Ltd.), No. 6, 2-chome, Fujimi-cho, Chiyoda-ku, Tokyo, Japan.

PUBLISHED BY PACIFIC JOURNAL OF MATHEMATICS, A NON-PROFIT CORPORATION

The Supporting Institutions listed above contribute to the cost of publication of this Journal, but they are not owners or publishers and have no responsibility for its content or policies. 


\section{Pacific Journal of Mathematics}

\section{Vol. 15, No. $2 \quad$ October, 1965}

Patrick Robert Ahern, On the generalized F. and M. Riesz theorem......... 373

A. A. Albert, On exceptional Jordan division algebras ................ 377

J. A. Anderson and G. H. Fullerton, On a class of Cauchy exponential

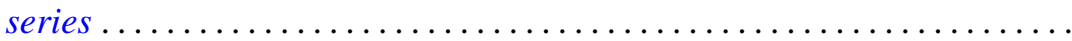

Allan Clark, Hopf algebras over Dedekind domains and torsion in

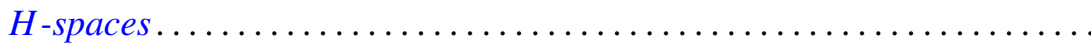

John Dauns and D. V. Widder, Convolution transforms whose inversion functions have complex roots .............................

Ronald George Douglas, Contractive projections on an $\mathrm{L}_{1}$ space ..........

Robert E. Edwards, Changing signs of Fourier coefficients ...............

Ramesh Anand Gangolli, Sample functions of certain differential processes on symmetric spaces .....................................

Robert William Gilmer, Jr., Some containment relations between classes of

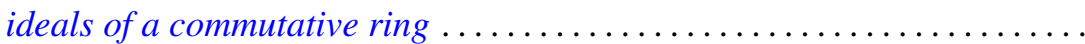

Basil Gordon, A generalization of the coset decomposition of a finite

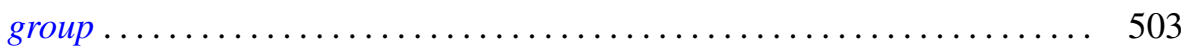

Teruo Ikebe, On the phase-shift formula for the scattering operator....... 511

Makoto Ishida, On algebraic homogeneous spaces ................ 525

Donald William Kahn, Maps which induce the zero map on homotopy ........ 537

Frank James Kosier, Certain algebras of degree one ................. 541

Betty Kvarda, An inequality for the number of elements in a sum of two sets of lattice points.................................

Jonah Mann and Donald J. Newman, The generalized Gibbs phenomenon for regular Hausdorff means. .

Charles Alan McCarthy, The nilpotent part of a spectral operator. II . ...

Donald Steven Passman, Isomorphic groups and group rings ...

R. N. Pederson, Laplace's method for two parameters .....

Tom Stephen Pitcher, A more general property than domination for sets of probability measures .............................

Arthur Argyle Sagle, Remarks on simple extended Lie algebras. .

Arthur Argyle Sagle, On simple extended Lie algebras over fields of

characteristic zero.

Tôru Saitô, Proper ordered inverse semigroups ...........

Oved Shisha, Monotone approximation

Indranand Sinha, Reduction of sets of matrices to a triangular form

Raymond Earl Smithson, Some general properties of multi-valued

functions .................................

John Stuelpnagel, Euclidean fiberings of solvmanifolds .... 